\title{
Pulse pressure, aortic regurgitation and carotid atherosclerosis: a comparison between hypertensives and normotensives
}

\author{
T-C SU, ${ }^{1,2}$ K-L CHIEN, ${ }^{1,3}$ J-S JENG,${ }^{4}$ C-J CHANG,${ }^{5}$ H-C HSU, ${ }^{1}$ M-F CHEN, ${ }^{1}$ F-C SUNG ${ }^{6}$ Y-T LEE ${ }^{1}$ \\ Department of Internal Medicine, ${ }^{1}$ National Taiwan University Hospital, Institute of Occupational Medicine and Industrial \\ Hygiene, ${ }^{2}$ Graduate Institute of Preventive Medicine, ${ }^{3}$ College of Public Health, National Taiwan University, Department of \\ Neurology, ${ }^{4}$ Department of Clinical Medicine, ${ }^{5}$ National Taiwan University Hospital, Taipei, Institute of Environmental \\ Health, ${ }^{6}$ China Medical University, Taichung, Taiwan
}

\section{SUMMARY}

Pulse pressure (PP) has often been associated with cardiovascular morbidity and mortality. Patients with aortic regurgitation (AR) often have increased PP. The aim of this study is to investigate the associations among PP, AR and extracranial carotid artery (ECCA) carotid atherosclerosis (CA) in hypertensives and age- and sex-matched normotensives. Two hundred and sixty-three hypertensive patients and 270 normotensive subjects from the Chin-Shan Community Cardiovascular Cohort participated in this study in 1996. CA, expressed as maximal common carotid artery intima-media thickness (IMT) $\geq 75$ th percentile and ECCA plaque score $>6$, was measured using highresolution B-mode ultrasonography. The presence of AR was assessed by echocardiography, and their relationships with CA were evaluated. Results showed measurements of
CA significantly associated with increased PP. Presence of AR associated with CA, but this relationship was attenuated after controlling for age. Multivariate logistic regression analyses revealed that an ECCA score $>6$ significantly increase the risk in conjunction with $\mathrm{PP}$, age and smoking in hypertensives. Correspondingly, CA increased with age, smoking and left ventricular hypertrophy on electrocardiography but not PP in normotensives. In conclusion, higher $\mathrm{PP}$ is strongly associated with CA in patients with hypertension. In terms of risk stratification, PP is more important in hypertensives than in normotensives which seem to imply that pulsatile haemodynamic component of BP is crucial in association with atherosclerosis.

Keywords: Pulse pressure; aortic regurgitation, carotid atherosclerosis; hypertension

(ㄷ) 2006 Blackwell Publishing Ltd

\section{INTRODUCTION}

There is a body of evidence associating pulse pressure (PP) with cardiovascular (CV) morbidity and mortality in the general population $(1,2)$. Stroke events and CV mortality also rise with increased PP $(3,4)$. The pulsatile component of blood pressure (BP) is mainly contributed by cardiac output and total peripheral resistance, which is expressed as PP. Increased PP has also been related to increased carotid intima-media thickness (IMT) and extracranial carotid artery (ECCA) carotid atherosclerosis (CA) (5-7). However, what remains controversial is whether $\mathrm{PP}$ predicts $\mathrm{CV}$ disease risk independently of either systolic BP (SBP) or diastolic BP (DBP) (8). The 1999 WHO-ISH report suggests more direct evidence about the risks associated with $\mathrm{BP}$ and other risk

Correspondence to:

Yuan-Teh Lee, MD, PhD, Emeritus Professor, National Taiwan University College of Medicine, No. 7, Chung-Shan South Road, Taipei 100, Taiwan

Tel.: + 886223562830

Fax: + 886223959922

Email: ytlee@ha.mc.ntu.edu.tw factors, and more observational epidemiological studies in Asian populations were encouraged. Furthermore, CA evaluation as an important prognostic surrogate endpoint was also suggested (8). Recently, high normal BP was shown to increase the risk of $\mathrm{CV}$ diseases in the Framingham Heart Study (9), which also raised the urgency of identifying subjects at higher risk of CV diseases in normotensive subjects.

It is well known that PP increases with age. There are other diseases where PP increases significantly, of which aortic regurgitation $(\mathrm{AR})$ is common in older population, which is attributed to a combination of forward stroke volume and regurgitant volume resulting in elevation of the aortic systolic pressure, and thus, the PP is considerably increased (10). Thus, in addition to age, the determinants of PP in these high-risk patients deserved our attention.

We have documented that hypertension plays a major role in the pathogenesis of CA and a dose-response relationship exists between hypertension status and CA (11). However, whether PP predisposes additional effects on the development of CA beyond hypertension status is unclear. Several studies show that PP was an important predictor for CV 
complications $(2,12-14)$. The aim of this study is to investigate the associations among PP, AR and ECCA atherosclerosis in subjects with hypertension and their normotensive controls.

\section{SUBJECTS AND METHODS}

\section{Study Subjects}

Since 1990, the Chin-Shan Community Cardiovascular Cohort (CCCC) study has been established as a longitudinal, prospective community-based surveillance on coronary artery disease (CAD), cerebrovascular diseases (CVD) and related atherosclerosis-risk factors. There were 3602 participants aged 35 years and above, residing in the Chin-Shan community, 20 miles North of Taipei, Taiwan, receiving CV assessments (11,15-17). The patients and control subjects in this study were recruited from the CCCC study cohort as previously reported (11). This study was conducted from July to December 1996, with 1802 individuals randomly selected as the study population. Of these, 451 subjects were classified as hypertension cases. From the rest of the 1351 normotensives, 451 were randomly selected by frequency matching for gender and age. A total of 263 hypertensives and 270 normotensive controls received the carotid ultrasonography and echocardiographic evaluations throughout this study. The CVrisk factors and demographic characteristics between responder and non-responder were similar.

\section{Assessment of Vascular-risk Factors}

Hypertension was defined according to the criteria established by the Fifth Report of the Joint National Committee on Prevention, Detection, Evaluation, and Treatment of High Blood Pressure (JNC V). Subjects with SBP higher than $140 \mathrm{mmHg}$ and/or DBP higher than $90 \mathrm{mmHg}$ or receiving antihypertension agents were considered hypertensive. BP measurements were performed with mercury sphygmomanometer in a standardised fashion. BP was recorded using the mean of two measurements taken after 5 min of rest in the supine position. Systolic and diastolic blood pressures were defined according to Korotkoff sounds I and V. PP was defined as the mean of two measurements of difference between systolic BP and diastolic BP. In this study, for the estimation of the risk of CA between different PP categories, PP was arbitrarily stratified as $\geq 60,50-59$ and $<50 \mathrm{mmHg}$.

DM was defined as fasting serum glucose $\geq 140 \mathrm{mg} / \mathrm{dl}$ in at least two different measurements and/or a history of use of medication. Left ventricular hypertrophy (LVH) was diagnosed from the electrocardiogram (ECG) according to the criteria defined in the Physician's Guide to Marquette Electronics Resting ECG.

\section{ECCA Ultrasonographic Measurements}

Carotid end-organ disease was assessed by the maximal common carotid artery (CCA) IMT and by two indices of atherosclerosis; the ECCA plaque score and the maximal ECCA stenosis. A Hewlett-Packard SONO 1500 ultrasound system, equipped with a $7.5 \mathrm{MHz}$ real-time B-mode scanner and a 5.6 MHz pulsed-Doppler mode scanner, was used for the evaluation. The examination protocol was the same as previously reported (11). For subsequent off-line analysis, all scans were recorded on super-VHS videotape.

The maximal IMT on the CCA proximal to the carotid bifurcation was obtained bilaterally. The CCA1 and CCA2 are points located $0-1 \mathrm{~cm}$ and $1-2 \mathrm{~cm}$ on the CCA distal from the carotid bifurcation, respectively. The intraclass correlation coefficients (ICCR) of the interobserver were 0.86 0.93 and those of the intraobserver were $0.70-0.87$ for both sides of the CCA IMT measurements (18).

The plaque scoring quantified method has been described in the past $(11,18,19)$. In brief, carotid artery segments, including the proximal and distal CCA $(>20 \mathrm{~mm}$ and 0-20 $\mathrm{mm}$ distal to the bulb bifurcation, respectively), bulb, internal carotid artery and external carotid artery, were examined bilaterally. The plaque score was computed by summing the plaque grades at 10 segments of the ECCA. Reproducibility of the ECCA plaque scoring was proved good agreement with a kappa value of 0.70 (19).

\section{Echocardiographic Assessments}

A Hewlett-Packard SONO 2500 ultrasound system, equipped with a $2.5 / 2.0 \mathrm{MHz}$ phase-array transducer, was used for the echocardiographic studies. All subjects were lying in the left lateral decubitus position to assure the standardised measurement. Observers were blind to the CV-risk factors and clinical condition. All pictures were taken at the end of expiratory phase and recorded on a super-VHS videotape for the further off-line analysis. The methods of echocardiographic measurements had been described before (20). The ICCR for interobserver variability of measurement were $0.70-0.85$ (20). The grade of AR was identified as methods from Cooper et al. (21). AR of Grade 1 was defined as a diastolic turbulent flow in the LV, with a jet $<20 \%$ of aortic annular diameter (AAD) and extending less than half the distance to the tip of anterior mitral leaflet (AML); Grade 2 AR by jet filling $20-40 \%$ of AAD and extending up to the tip of AML and grade $>3$, if a jet $>40 \%$ of AAD and extending beyond the tip of AML in at least three consecutive colour Doppler frames.

\section{Lipid and Lipoprotein Assays}

Blood samples of $9-12 \mathrm{~h}$ of overnight fasting for lipid and glucose determination were drawn from an antecubital vein with the patient in a seated position. Serum levels of lipid profiles, including total cholesterol, low-density lipoprotein cholesterol (LDL-C), high-density lipoprotein cholesterol 
(HDL-C) and triglyceride, were analysed in a central laboratory and have been described previously $(11,15-17)$.

\section{Statistical Analyses}

Because hypertension status was defined according to cut point of systolic and diastolic BP values and hypertension history, the presentations of PP and CA do not be necessarily limited in binary discipline that dividing in hypertensives or normotensives. Because the values of PP were strongly related with hypertension status, their relationships with CA can be realised by all participants. For CA evaluation in this study, either hypertensives or normotensives were considered altogether. In summary, the extents of CA were compared according to different grades of PP and AR in all participants, combining hypertensives and normotensives.

This is a nested case-control study, and our primary hypothesis was to test the association between PP gradient and CA severity. From data in Table 2, we applied ANOVA to estimate the power of the study as follows. When the total sample size across the three groups is 533, distributed across the groups as specified, a one-way ANOVA will have 99\% power to detect at the 0.050 level and an effect size of 0.800 among three different CCA thicknesses.

Clinical features and CV-risk factors of the study subjects were compared by hypertensives and normotensives. Continuous variables are expressed as the mean $\pm 1 \mathrm{SD}$, and binary data was expressed as percentage. Both student's $t$-test and ANOVA were used to make comparisons between these groups. The association between PP and CA was evaluated by ANOVA according to the average carotid artery IMT measurements at CCA1 and CCA2 on both sides and average scores of ECCA plaques after adjustment for age and gender. The values of crude and after adjustment for age of average CCA IMT and CCA scores were compared also by grade of AR.

In the association analysis, we estimate the relative risk of $\mathrm{PP}$ in hypertensives and normotensives. The strength of associations between PP and CA and other potential-risk factors were estimated in terms of odds ratios in the $95 \%$ confidence interval using conditional logistic regression analysis. Results with an IMT $\geq 75$ th percentile and an ECCA score $>6$ were considered to be indicators of significant CA in performing these analyses.

\section{RESULTS}

In Table 1, subjects with hypertension had significant higher pressure in different BP components [SBP, DBP, PP and mean artery pressure (MAP)], higher LDL-C levels, lower HDL-C levels and a higher body mass index compared with normotensives. More prevalent in CVD, CAD, DM and LVH on ECG were found in hypertensives. Among patients with hypertensives, $8.33 \%$ were CVD and $5.07 \%$ were CAD. In contrast, the corresponding prevalence of CVD and CAD were 1.56 and $2.33 \%$ in the normotensives, respectively.

Table 1 Clinical and cardiovascular risk factors in hypertensives and normotensives

\begin{tabular}{lccr}
\hline Characteristics & Hypertensives $(n=276)$ & Normotensives $(n=257)$ & $p$ value \\
\hline Male, \% & 43.48 & 42.02 & 0.793 \\
Age & $65.1 \pm 9.8$ & $64.1 \pm 9.4$ & 0.214 \\
Systolic BP, mmHg & $141.2 \pm 18.7$ & $122.2 \pm 16.5$ & $<0.001$ \\
Diastolic BP, mmHg & $83.8 \pm 10.0$ & $76.4 \pm 9.0$ & $<0.001$ \\
Pulse pressure, mmHg & $57.3 \pm 12.9$ & $45.8 \pm 10.4$ & $<0.001$ \\
Mean artery pressure, mmHg & $102.9 \pm 12.1$ & $91.6 \pm 11.0$ & $<0.001$ \\
Body mass index, kg/m 2 & $25.0 \pm 3.6$ & $23.7 \pm 4.1$ & $<0.001$ \\
Smoke current, \% & 19.57 & 21.01 & 0.747 \\
Alcohol current, \% & 19.57 & 24.51 & 0.175 \\
Cholesterol, mg/dl & $223.42 \pm 45.48$ & $215.95 \pm 39.23$ & $0.119^{*}$ \\
HDL-C, mg/dl & $39.9 \pm 11.1$ & $44.3 \pm 11.8$ & $<0.001$ \\
LDL-C, mg/dl & $148.8 \pm 45.1$ & $135.0 \pm 40.0$ & $<0.001$ \\
LVH on ECG, \% & 13.41 & 6.23 & 0.006 \\
Diabetes mellitus, \% & 21.4 & 13.6 & $<0.001$ \\
Aortic regurgitation & $68(25.86 \%)$ & $42(15.59 \%)$ & $<0.001$ \\
Antihypertensive medications, \% & 47.1 & - & - \\
Cerebrovascular disease, \% & 8.33 & 1.56 & $<0.001$ \\
Coronary heart disease, \% & 5.07 & 2.33 & 0.097 \\
LV systolic dysfunction, \% & 2.17 & 1.17 & $0.507 \dagger$ \\
\hline
\end{tabular}

HDL-C, high-density lipoprotein cholesterol; LDL-C, low-density lipoprotein cholesterol; LVH on ECG, left ventricular hypertrophy diagnosed by electrocardiogram; LV systolic dysfunction, defined as LV ejection fraction $<50 \% .{ }^{*}$ Wilcoxon rank sum test was used to compare the difference between two groups. $\dagger$ Fisher's exact test was used to test the difference. 
Among hypertensives, $47.1 \%$ of patients received antihypertensive medications. LV systolic dysfunction, defined as LV ejection fraction $<50 \%$, was found with six subjects $(2.17 \%)$ in hypertensives compared with three subjects $(1.17 \%)$ in normotensives.

Average CCA IMT measurements and ECCA plaque score after adjustment for age and gender at different carotid locations all showed significant trends $(\mathrm{p}<0.001)$ of increment as graded by PP (Table 2). The overall average IMT at RCCA increased progressively from $0.91 \pm 0.02 \mathrm{~mm}$ for $\mathrm{PP}<50 \mathrm{mmHg}$ to $0.96 \pm 0.01 \mathrm{~mm}$ for $\mathrm{PP}=50-59 \mathrm{mmHg}$ and then to $1.06 \pm 0.02 \mathrm{~mm}$ for $\mathrm{PP}>60 \mathrm{mmHg}$. The average plaque score was $1.42 \pm 0.21$ while $\mathrm{PP}<50 \mathrm{mmHg}$ and increased to $1.84 \pm 0.25$ while the corresponding $\mathrm{PP}=50-59 \mathrm{mmHg}$ and $2.71 \pm 0.26$ at $\mathrm{PP} \geq 60 \mathrm{mmHg}$.

The measurements of average CCA IMT and ECCA plaque score also showed significant trends $(\mathrm{p}<0.001)$ of progression as graded by extent of AR (Table 3 ). However, the trends of IMT increment and plaque-score progression were attenuated while adjusting for age.

We evaluated the true relationship between higher PP and $\mathrm{CA}$ in hypertensives and normotensives. Table 4 summarises the results of the univariate logistic regression analysis. In addition to age, PP and current smoking were strongly associated with thicker IMT and significant CA. However, in normotensive group, age, PP, male gender and smoking were associated with thicker IMT. And age and LVH on ECG were related with ECCA scores $>6$.

In Table 5, multivariate logistic regression models revealed that PP, male gender, smoking and age significantly increases the risk of thicker IMT in hypertensives; however, only age increased the above risk in normotensives. Furthermore, an ECCA score $>6$ significantly increases the risk in conjunction with PP, age and smoking in hypertensives. On the contrast, age, smoking and LVH on ECG, but not PP, increased the risk for significant CA in normotensives. In Table 6, SBP and age were the two major determinants for increased PP. Other factors associated with an increase in PP were DM and LVH on ECG.

\section{DISCUSSIONS}

Hypertension has been demonstrated to be the major determinant of CA (11), with a high impact on stroke events with relative risks of 2.5-3.0 after an 8-year follow-up in this cohort study (22). The present study is well in line with other findings in the association between CA and PP (5-7). Also, we demonstrate that a dose-response relationship exists between PP and CA. Presence of AR was associated with CA, but the relationship was not evident while age was controlled. And the major determinants of PP were age and SBP but not the presence of AR. This study is the first to demonstrate the dose-response effect of PP for thicker IMT and CA in Asian population. And we provided for the first time that the positive association between AR and CA.

PP is determined by the peripheral resistance and elastic properties of the arterial wall, which are associated with atherosclerosis and CV-risk factors. Wide PP also increases the haemodynamic barotrauma on the vascular wall and subsequently predisposes detrimental effects on the endothelium. Increased PP may promote atherosclerosis through pulsatile blood flow, or cyclic strain, which has been shown to affect endothelial cell gene expression and function.

In one meta-analysis, based on older hypertensive patients, PP but not MAP is the major determinant of CV risk (11). Data from the Physicians' Health Study found that average SBP, DBP and MAP strongly predict CVD among younger men, whereas either average SBP or PP predicts CVD among older men (23). The Framingham Heart Study showed that, with increasing age, there was a gradual shift from DBP to SBP at age below 60 years and then to PP at age 60 years or above as predictors of CHD risk (24). The fall in DBP after age 60 years old signals a preponderance of large artery stiffness as the cause of a further rise in SBP. Thus, PP was

Table 2 CCA IMT measurements in different location and ECCA plaque score by grade of pulse pressure

\begin{tabular}{|c|c|c|c|c|}
\hline \multirow[b]{2}{*}{ IMT location } & \multicolumn{3}{|c|}{ Pulse pressure, $m m H g^{*}$} & \multirow[b]{2}{*}{$p$ value for trend } \\
\hline & $<50(n=223)$ & $50-59(n=158)$ & $\geq 60(n=152)$ & \\
\hline RCCA1 & $0.94 \pm 0.02$ & $1.00 \pm 0.02$ & $1.11 \pm 0.02$ & $<0.001$ \\
\hline RCCA2 & $0.88 \pm 0.02$ & $0.92 \pm 0.02$ & $1.01 \pm 0.02$ & $<0.001$ \\
\hline RCCA & $0.91 \pm 0.02$ & $0.96 \pm 0.02$ & $1.06 \pm 0.02$ & $<0.001$ \\
\hline LCCA1 & $0.94 \pm 0.02$ & $0.95 \pm 0.02$ & $1.09 \pm 0.02$ & $<0.001$ \\
\hline LCCA2 & $0.87 \pm 0.01$ & $0.92 \pm 0.02$ & $1.00 \pm 0.02$ & $<0.001$ \\
\hline LCCA & $0.91 \pm 0.02$ & $0.93 \pm 0.02$ & $1.04 \pm 0.02$ & $<0.001$ \\
\hline ECCA plaque score & $1.42 \pm 0.21$ & $1.84 \pm 0.25$ & $2.71 \pm 0.26$ & $<0.001$ \\
\hline
\end{tabular}

CCA, common carotid artery; CCA, mean IMT of both RCCA and LCCA; IMT, intima-media thickness; LCCA, mean IMT of both LCCA1 and LCCA2; RCCA, mean IMT of both RCCA1 and RCCA2. Values are least square mean \pm SE, age and sex adjusted. ${ }^{*}$ Thickening in mm. 
Table 3 Common carotid artery intima-media thickness (IMT) and carotid atherosclerosis by grade of aortic regurgitation

\begin{tabular}{lllll}
\hline & \multicolumn{3}{c}{ Aortic regurgitation } \\
\cline { 2 - 4 } Characteristics & Absence $(n=423)$ & Grade $1(n=81)$ & Grade $\geq 2(n=29)$ & p value for trend \\
\hline IMT $\geq 75$ th percentile (\%) & 26.0 & 37.0 & 41.4 & 0.006 \\
IMT, mm* & $0.94 \pm 0.22$ & $1.01 \pm 0.24$ & $1.08 \pm 0.32$ & 0.002 \\
IMT $\mathrm{mm}^{\dagger}$ & $0.95 \pm 0.01$ & $0.99 \pm 0.02$ & $1.01 \pm 0.25$ & 0.137 \\
ECCA score $>6(\%)$ & 8.0 & 12.4 & 20.7 & 0.008 \\
ECCA plaque score* & $1.70 \pm 3.24$ & $2.51 \pm 3.74$ & $3.34 \pm 4.32$ & 0.011 \\
ECCA plaque score $\dagger$ & $1.80 \pm 0.15$ & $2.27 \pm 0.35$ & $2.52 \pm 0.60$ & 0.244 \\
Carotid stenosis $\geq 50 \%(\%)$ & 3.3 & 4.9 & 6.9 & 0.170 \\
\hline
\end{tabular}

*Values are expressed as mean \pm standard deviation; †Least square Mean \pm SE, age adjusted

Table 4 Univariate logistic regression model for thicker intima-media thickness (IMT) or moderate to severe carotid atherosclerosis

\begin{tabular}{llllll}
\hline & \multicolumn{4}{c}{ Odds ratio (95\% confidence interval) } \\
\cline { 2 - 3 } & \multicolumn{1}{l}{ Hypertensives } & & & Normotensives & \\
\cline { 2 - 3 } & $I M T \geq 75$ th percentile & ECCA score $>6$ & & IMT $\geq 75$ th percentile & ECCA score $>6$ \\
\hline PP, mmHg & $1.04(1.02-1.06) \ddagger$ & $1.05(1.02-1.08) \ddagger$ & & $1.03(1.00-1.06)^{*}$ & $1.02(0.96-1.07)$ \\
MED & $1.42(0.88-2.30)$ & $1.36(0.70-2.65)$ & & - & - \\
Age, year & $1.08(1.05-1.11) \ddagger$ & $1.09(1.05-1.14) \ddagger$ & & $1.10(1.05-1.15) \ddagger$ & $1.18(1.08-1.30) \ddagger$ \\
Male & $1.45(0.89-2.34)$ & $1.82(0.93-3.55)$ & & $2.61(1.27-5.35) \dagger$ & $2.13(0.59-7.75)$ \\
Smoking & $2.34(1.28-4.30) \dagger$ & $2.53(1.22-5.24) \dagger$ & & $2.74(1.30-5.78) \dagger$ & $2.63(0.71-9.67)$ \\
Alcohol & $0.69(0.37-1.28)$ & $0.67(0.27-1.68)$ & & $0.99(0.44-2.22)$ & $2.12(0.58-7.78)$ \\
DM & $1.76(1.00-3.11)$ & $1.32(0.62-2.82)$ & & $1.58(0.60-4.18)$ & $0.84(0.10-6.83)$ \\
HDL, mg/dl & $0.99(0.97-1.02)$ & $1.00(0.97-1.03)$ & & $0.98(0.95-1.01)$ & $0.94(0.88-1.01)$ \\
LDL, mg/dl & $1.00(1.00-1.01)$ & $1.00(0.99-1.01)$ & & $1.00(0.99-1.01)$ & $1.00(0.98-1.01)$ \\
LVH on ECG & $1.34(0.67-2.68)$ & $2.08(0.90-4.81)$ & & $0.84(0.18-3.86)$ & $7.71(1.79-33.33) \dagger$ \\
\hline
\end{tabular}

DM, diabetes mellitus; HDL, high-density lipoprotein cholesterol; LDL, low-density lipoprotein cholesterol; LVH on ECG, left ventricular hypertrophy diagnosed by electrocardiography; MAP, mean artery pressure; MED, antihypertensive medication; PP, pulse pressure. ${ }^{*} \mathrm{p}$ value $<0.05 ; \nmid \mathrm{p}$ value $<0.01 ; \ddagger \mathrm{p}$ value $<0.005$.

Table 5 Multivariate logistic regression model for thicker intimamedia thickness (IMT) or moderate to severe carotid atherosclerosis

\begin{tabular}{lll}
\hline & $I M T \geq 75$ th percentile & ECCA score $>6$ \\
\hline Hypertensives & & \\
$\quad$ PP, mmHg & $1.03(1.01-1.06) \dagger$ & $1.04(1.01-1.07) \dagger$ \\
MED & $1.06(0.60-1.86)$ & $1.08(0.50-2.33)$ \\
Age, year & $1.07(1.04-1.11) \ddagger$ & $1.09(1.04-1.14) \ddagger$ \\
Male & $1.49(0.74-2.99)$ & $1.90(0.74-4.90)$ \\
Smoking & $3.80(1.57-9.23) \ddagger$ & $3.22(1.13-9.23)^{*}$ \\
Alcohol & $0.39(0.16-0.93) \dagger$ & $0.41(0.13-1.28)$ \\
LVH on ECG & $0.81(0.35-1.86)$ & $1.06(0.39-2.87)$ \\
Normotensives & & \\
PP, mmHg & $1.01(0.98-1.05)$ & $0.98(0.91-1.06)$ \\
Age, year & $1.09(1.04-1.15) \ddagger$ & $1.24(1.08-1.41) \ddagger$ \\
Male & $2.38(0.95-5.95)$ & $0.34(0.05-2.51)$ \\
Smoking & $2.43(0.94-6.31)$ & $6.79(1.01-15.046)^{*}$ \\
Alcohol & $0.73(0.27-1.98)$ & $4.96(0.84-19.34)$ \\
LVH on ECG & $0.38(0.07-2.00)$ & $6.92(1.05-15.67)^{*}$ \\
\hline
\end{tabular}

LVH on ECG, left ventricular hypertrophy on electrocardiography; MED, antihypertensive medication; PP, pulse pressure. Models were after adjustment for above variables and diabetes mellitus, body mass index, high-density lipoprotein and low-density lipoprotein cholesterol. ${ }^{*} \mathrm{p}$ value $<0.05$; †p value $<0.01 ;$ ‡p value $<0.005$.
Table 6 Multiple lineal regression analysis for the determinants of pulse pressure

\begin{tabular}{lcr}
\hline Variable & Parameter estimate & p value \\
\hline Intercept & -35.6 & $<0.0001$ \\
Systolic BP & 0.56 & $<0.0001$ \\
Aortic regurgitation & & \\
$\quad$ Grade 1 & 1.02 & 0.1831 \\
$\quad$ Grade $\geq 2$ & 1.44 & 0.2327 \\
Age & 0.22 & $<0.0001$ \\
Male & -0.45 & 0.5043 \\
Body mass index & -0.27 & 0.9415 \\
Smoke current & -0.27 & 0.7334 \\
Alcohol current & 0.35 & 0.6407 \\
Triglyceride & -0.006 & 0.1362 \\
LDL-C & -0.005 & 0.4200 \\
Diabetes mellitus & 2.07 & 0.0048 \\
LVH on ECG & 1.87 & 0.0439 \\
\hline
\end{tabular}

BP, blood pressure; ECG, electrocardiogram; LDL-C, low-density lipoprotein cholesterol; LVH, left ventricular hypertrophy. Adjusted $\mathrm{r}^{2}=0.79$. 
found to be important in association with CA in this study, which may be an age-related difference, because the average age of participants was 65 years.

We have demonstrated 24-h average SBP and DBP as positive and negative important determinants of $\mathrm{CA}$, respectively, which implied that 24-h average PP significantly increased CA (25). This study identified SBP as the major determinant of PP. Hypertension, especially in wide PP, increases cyclic stretch during systole, which may amplify the degeneration of the vessel wall, leading to earlier and more severe arterial stiffness and atherosclerosis. This study found a positive association between echocardiographic AR and CA. However, the associations were attenuated by age. In addition, we can not find $\mathrm{AR}$ as a significant determinant for $\mathrm{PP}$ in multiple regression analysis. Thus, PP-increased CA regardless of age and other $\mathrm{CV}$ factors in hypertensives in our study provide an evidence of true detrimental CV effect of pulsatile BP components.

As in previous studies $(6,25,26)$, this study found that age and male gender are two major determinants of CA. Cigarette smoking is significantly related to increase carotid artery IMT and carotid stenosis $(11,27)$. Smoking has a consistent detrimental effect on CA independent of hypertension status in this study. LVH on ECG has been associated with atherosclerosis of carotid arteries (26). The findings of LVH on ECG as a significant predictor for thicker IMT and CA in the normotensives but not in the hypertensives in current study indicated that some causes of LVH are independent of hypertension, which may play an important role in association with CA.

The present study links PP, age and smoking as the most prominent risk factors for thicker IMT and clinically for CA. In addition, this study demonstrated that PP is the important factor in association with CA in patients with hypertension. This finding indicates that increased PP, an indicator of large artery stiffness, may increase the risk of atherosclerosis.

\section{Study Limitations}

This study is limited by its cross-sectional design. Because the higher PP may be produced by advanced atherosclerosis, the causal relationship between increased PP and CA cannot be concluded in this design. As the strong associations among higher PP, presence of AR and CA in older population, and the interrelationship made the exploration of true association between PP and CA more complex.

\section{CONCLUSIONS}

The present study showed PP to be an additional risk factor for CA among individuals with hypertension. Our present findings may have important clinical implications in terms of risk assessment and potential-risk reduction indices using simple PP measurements. Optimal control of hypertension, especially in patients with wide $\mathrm{PP}$, is very important for the prevention of atherosclerotic disease.

\section{ACKNOWLEDGEMENTS}

We thank Ms Ching-Chu Chien and the cardiologists at National Taiwan University Hospital for their assistance. This study was partly sponsored by the Power Development Foundation of Taiwan Power Company (1996) and grants from the National Science Council (NSC\#90-2314-B-002117 and NSC\#90-2320-B-002-178) in Taiwan.

\section{REFERENCES}

1 Franklin SS, Khan SA, Wong ND, Larson MG, Levy D. Is pulse pressure useful in predicting risk for coronary heart disease? The Framingham Heart Study. Circulation 1999; 100: 354-60.

2 Benetos A, Safar M, Rudnichi A et al. Pulse pressure - a predictor of long-term cardiovascular mortality in a French male population. Hypertension 1997; 30: 1410-5.

3 Domanski MJ, Davis BR, Pfeffer MA, Kastantin M, Mitchell GF. Isolated systolic hypertension - prognostic information provided by pulse pressure. Hypertension 1999; 34: 375-80.

4 Benetos A, Rudnichi A, Safar M, Guize L. Pulse pressure and cardiovascular mortality in normotensive and hypertensive subjects. Hypertension 1998; 32: 560-4.

5 Zureik M, Touboul PJ, Bonithon-Kopp C et al. Cross-sectional and 4-year longitudinal associations between brachial pulse pressure and common carotid intima-media thickness in a general population. Stroke 1999; 30: 550-5.

6 Salonen R, Salonen JT. Determinants of carotid intima-media thickness: a population-based ultrasonography study in eastern Finnish men. J Intern Med 1991; 229: 225-31.

7 Sutton-Tyrrell K, Belle SH, Weber MA, Kuller LH. The importance of pulsatile components of hypertension in predicting carotid stenosis in older adults. J Hypertens 1997; 15: 1143-50.

81999 World Health Organization-International Society of Hypertension guidelines for the management of hypertension. Guidelines Subcommittee. J Hypertens 1999; 17: 151-83.

9 Vasan RS, Larson MG, Evans JC et al. Impact of high-normal blood pressure on the risk of cardiovascular disease. $N$ Engl J Med 2001; 345: 1291-7.

10 Carabello BA, Crawford FA Jr. Valvular heart disease. $N$ Engl J Med 1997; 337: 32-41.

$11 \mathrm{Su}$ TC, Jeng JS, Chien KL et al. Hypertension status is the major determinant of carotid atherosclerosis: a community-based study in Taiwan. Stroke 2001; 32: 2265-71.

12 Darné B, Girerd X, Safar M et al. Pulsatile versus steady components of blood pressure: a cross-sectional analysis and a prospective analysis on cardiovascular mortality. Hypertension 1989; 13: 392-400.

13 Gasowski J, Fagard RH, Staessen JA et al. Pulsatile blood pressure and components as predictor of mortality in hypertension: a meta-analysis of clinical trial control groups. J Hypertens 2002; 20: $145-51$. 
14 Lee YT, Lin RS, Sung FC et al. Chin-Shan Community Cardiovascular Cohort in Taiwan - baseline data and five-year follow-up morbidity and mortality. J Clin Epidemiol 2000; 53: $838-46$.

15 Torng PL, Su TC, Sung FC et al. Effects of menopause on intraindividual changes in serum lipids, blood pressure, and body weight: the Chin-Shan community cardiovascular cohort study. Atherosclerosis 2002; 161: 409-15.

16 Chien KL, Lee YT, Sung FC et al. Hyperinsulinemia and related atherosclerotic risk factors in the population at risk: a community-based study. Clin Chem 1999; 45: 838-46.

17 Su TC, Jeng JS, Chien KL et al. Measurement reliability of common carotid artery intima-media thickness by ultrasonographic assessment. J Med Ultrasound 1999; 7: 73-9.

18 Jeng JS, Chung MY, Yip PK et al. Extracranial carotid atherosclerosis and vascular risk factors in different types of ischemic stroke in Taiwan. Stroke 1994; 25: 1989-93.

19 Chen CC, Chung MY, Jeng JS et al. A scoring system for evaluation of the extent of extracranial carotid atherosclerosis with B-mode imaging. Acta Neurol Sin 1995; 4: 29-33.

20 Chien KL, Sung FC, Hsu HC et al. Left ventricular mass and correlated atherosclerotic risk factors in young adolescents: report from Chin-Shan community cardiovascular study in Taiwan. Atherosclerosis 2001; 155: 431-7.

21 Cooper JW, Nanda NC, Philpot EF et al. Evaluation of valvular regurgitation by color Doppler. JAm Soc Echocardiogr 1989; 2: 56-65.
22 Chien KL, Sung FC, Hsu HC et al. Apolipoprotein A-1 and B and stroke events in a community-based cohort in Taiwan: report of the Chin-Shan Community Cardiovascular Study. Stroke 2002; 33: 39-44.

23 Sesso HD, Stampfer MJ, Rosner B et al. Systolic and diastolic blood pressure, pulse pressure and mean arterial pressure as predictors of cardiovascular disease risk in men. Hypertension 2000; 36: 801-7.

24 Franklin SS, Larson MG, Khan SA et al. Does the relation of blood pressure to coronary heart disease risk change with aging? The Framingham Heart Study. Circulation 2001; 103: 1245-9.

25 Su TC, Lee YT, Chou S, Hwang WT, Chen CF, Wang JD. Twenty-four-hour ambulatory blood pressure and duration of hypertension as major determinants for intima-media thickness and atherosclerosis of carotid arteries. Atherosclerosis 2006; 184: 151-6. 1 June 2005 Epub.

26 O'Leary DH, Polak JF, Kronimal RA et al. Distribution and correlates of sonographic detected carotid artery disease in the Cardiovascular Health Study. Stroke 1992; 23: 1752-60.

27 Tell GS, Polak JF, Ward BJ et al. Relation of smoking with carotid artery wall thickness and stenosis in older adults. Circulation 1994; 90: 2905-8.

Paper received March 2005, accepted November 2005 